\title{
Picosecond 266-nm Multiphoton Laser Photolysis Studies on the Solvated Electron Formation Process in Water and Liquid Alcohols
}

\author{
HIROSHI MIYASAKA, HIROSHI MASUHARAT \\ and NOBORU MATAGA \\ Department of Chemistry, Faculty of Engineering Science, \\ Osaka University, Toyonaka, Osaka 560, Japan
}

\begin{abstract}
A picosecond 266-nm multiphoton laser photolysis method was applied to the investigation of the solvation process of electrons in water and in several pure liquid alcohols. The formation time of the solvated electron obtained by this method in each liquid was revealed to be shorter than that obtained by picosecond pulse radiolysis and by the picosecond two-photon ionization of the solute molecules. By comparing the present results with those obtained by other methods, the effects of the excitation energy, as well as the solvation sphere of the relatively large parent cation on the solvated electron formation process, were elucidated.
\end{abstract}

KEY WORDS: Picosecond 266-nm multiphoton laser photolysis; picosecond transient absorption spectroscopy; solvated electron formation process; water; liquid alcohols; geminate ion pair.

\section{INTRODUCTION}

The application of picosecond laser ultraviolet (UV) multiphoton excitation has made it possible to observe the picosecond dynamics of various processes in the higher excited state, such as ionization,

$\dagger$ Present address: Department of Polymer Science and Engineering, Kyoto Institute of Technology, Matsugasaki, Kyoto, 606, Japan. 
dissociation and other chemical reactions in the condensed phase. Note that the UV laser multiphoton absorption is equal to the excitation in the vacuum ultraviolet (VUV) region. By using this ingenious method, we have demonstrated the photoprimary processes in higher excited states, not only for aromatic molecular liquids ${ }^{1,2}$, but also for aliphatic ones such as liquid alkyl halides ${ }^{3}$ and liquid alkanes ${ }^{4}$, of which investigation in the picosecond time domain had not been performed because of the lack of an appropriate picosecond laser light source. From these investigations, it was revealed that the transient species produced via UV picosecond multiphoton absorption are very similar to those observed by means of picosecond pulse radiolysis. This result suggests that the ionization plays an important role in the picosecond UV multiphoton photolysis, since it is believed that the formation of the ionic species and their dynamic behaviour are the most important processes in the early events of the radiation chemical reactions. Hence, the comparison of the results obtained by picosecond UV multiphoton laser photolysis with those by pulse radiolysis with respect to the effect of the different excitation energy on the subsequent behavior of the charged species produced, as well as of excited states, will be very fruitful.

In this paper, we report the results of the picosecond 266-nm two-photon photolysis studies on water and several liquid alcohols. We have confirmed that, in these liquids, the solvated electron is produced by a two-photon absorption of the incident 266-nm pulse. The results will be compared with those obtained by picosecond pulse radiolysis $^{5-7}$ and those by picosecond laser photolysis using twophoton ionization of the solute molecules ${ }^{8-11}$. On the basis of this comparison, we will discuss the solvation process of the ejected electron, taking into consideration the effect of the incident excitation energy, the excess energy of the ejected electron and the solvation shell of tne parent cation. A preliminary result on the formation of the solvated electron in water and in a few pure alcohols observed by means of picosecond 266-nm multiphoton laser photolysis method, was given in a previous report ${ }^{12}$.

\section{EXPERIMENTAL}

A microcomputer-controlled picosecond laser photolysis system with a repetitive $\mathrm{Nd}^{3+}$ :YAG laser was used to measure transient absorption 
spectra. The details of this system have been reported elsewhere ${ }^{13,14}$. The samples were excited with a single $266 \mathrm{~nm}$ pulse with about $20 \mathrm{ps}$ FWHM and $0.5-1 \mathrm{~mJ}$ output power.

Deionized water was distilled once and redistilled with a distillation apparatus of a non-boiling type. $\mathrm{D}_{2} \mathrm{O}$ (Uvasol 99.95\%), methanol, ethanol, 1-propanol, 2-propanol, 1-butanol (Dotite Spectrosol), and ethylene glycol (Nakarai Special Guarantee) were used without further purification. 1-Pentanol, tert-butyl alcohol and isobutyl alcohol (Nakarai Special Guarantee) were distilled under reduced pressure before use. All the samples were deaerated by irrigating with the $\mathrm{N}_{2}$ stream and a Suprasil cell with $1 \mathrm{~cm}$ pathlength was used. Measurements were performed at $22 \pm 1^{\circ} \mathrm{C}$.

\section{RESULTS AND DISCUSSION}

Figure 1 shows the transient absorption spectra of $\mathrm{H}_{2} \mathrm{O}, \mathrm{D}_{2} \mathrm{O}$ and some pure liquid alcohols observed at $100 \mathrm{ps}$ after excitation with a pico-

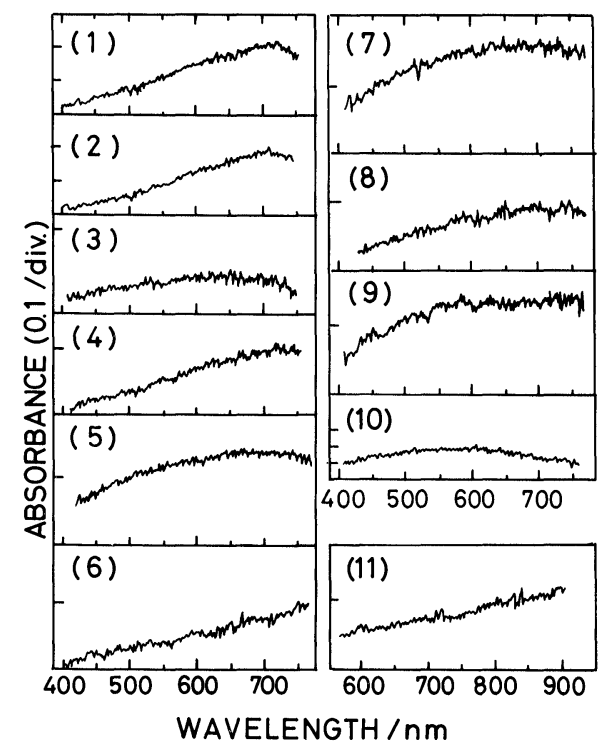

Figure 1 Transient absorption spectra of some pure liquids observed at $100 \mathrm{ps}$ after excitation with 266-nm picosecond laser pulse. (1) $\mathrm{H}_{2} \mathrm{O}$, (2) $\mathrm{D}_{2} \mathrm{O}$, (3) methanol, (4) ethanol, (5) 1-propanol, (6) 2-propanol, (7) 1-butanol, (8) 1-pentanol, (9) isobutyl alcohol, (10) ethylene glycol and (11) tert-butyl alcohol. 
second 266-nm laser pulse. All the spectra are very broad and structureless. No change in spectral shape or intensity was observed up to $3 \mathrm{~ns}$ in each case, suggesting that the lifetime of each transient is greater than tens of nanoseconds. These spectra could be assigned to the solvated electron in each solvent on the basis of good correlation in the spectral shape and its absorption maximum when comparing the present results and those measured by radiolysis ${ }^{5-7}$. From the excitation intensity dependence of the transient absorbance, it was found that these transient species were produced via a two-photon absorption process. Integrating these results with the fact that these liquids have no absorption at $266 \mathrm{~nm}$ in the ground state, simultaneous two-photon absorption of $266 \mathrm{~nm}$ laser light $(9.32 \mathrm{eV})$ is responsible for the ionization of water and pure liquid alcohols followed by the formation of solvated electron.

Figure 2 illustrates the time-resolved transient absorption spectra of liquid 1-propanol, showing that the spectrum at 40 ps shows relatively higher intensity at the longer wavelength regions compared with those at $20 \mathrm{ps}$. In general, the spectra immediately following excitation are different from those at later stages, because of the wavelength-

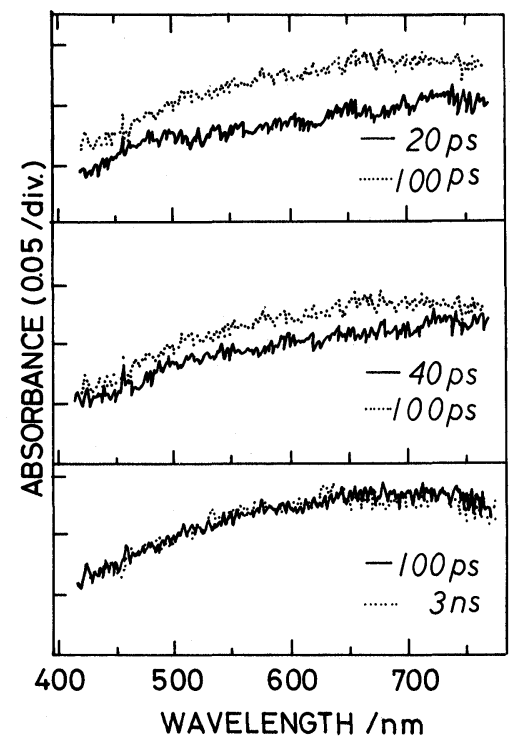

Figure 2 Time-resolved transient absorption spectra of pure 1-propanol obtained by excitation with picosecond 266-nm laser light. 
dependent distribution of the arrival time of the picosecond continuum at the sample position. According to our recent study ${ }^{14,15}$, the shorter the wavelength of the picosecond continuum, the later it reaches the sample. The difference of the arrival time between 450 and $650 \mathrm{~nm}$ is found to be about 10-15 ps. Because of this effect, a simple comparison of the spectrum immediately after excitation with that at a later stage is not appropriate. The above effect causes the absorbance of the spectra immediately after excitation to be stronger in the shorter wavelength region and we observe this effect around $450-500 \mathrm{~nm}$ in the spectrum at $20 \mathrm{ps}$. However, the spectra at delay times longer than $40 \mathrm{ps}$ indicate the growing-up of the spectra in whole regions of $400-750 \mathrm{~nm}$. Summarizing the above discussions, it can be concluded that the transient absorption spectra observed immediately after excitation in 1propanol include the contributions from the precursor species of the solvated electron which intrinsically has an absorption maximum in the infrared region. The same tendency was observed also in cases of primary alcohols such as ethanol, 1-butanol and 1-pentanol.

In order to estimate the formation time of solvated electrons, absorbance is plotted against delay time in Figure 3. Except from

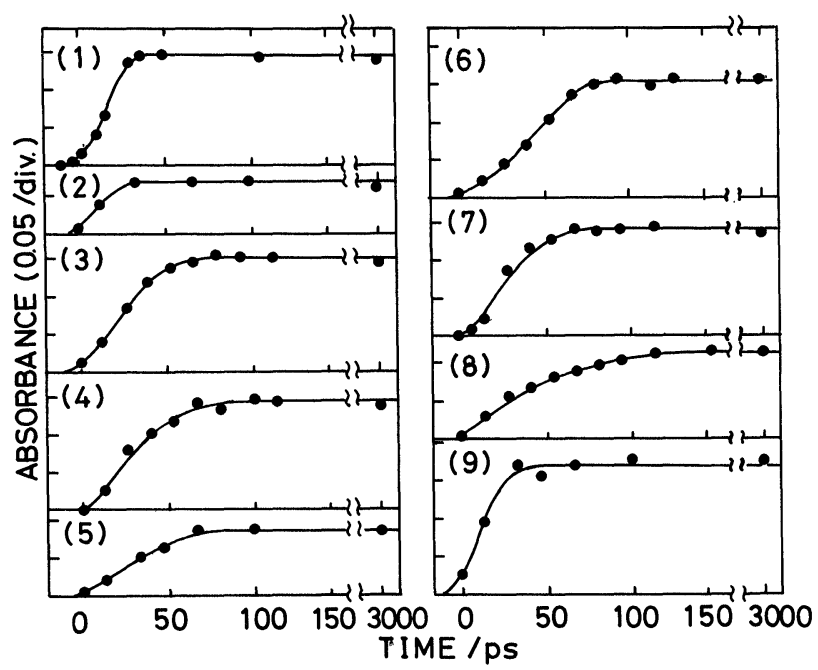

Figure 3 Rise curves of the transient absorbances of pure liquids. (1) $\mathrm{H}_{2} \mathrm{O}$, (2) methanol, (3) ethanol, (4) 1-propanol, (5) 2-propanol, (6) 1-butanol, (7) 1-pentanol, (8) tert-butyl alcohol and (9) ethylene glycol. 
2-propanol and tert-butyl alcohol, the peak absorbance of each spectrum is analyzed, while the absorbance at $750 \mathrm{~nm}$ and that at $900 \mathrm{~nm}$ are plotted for 2-propanol and for tert-butyl alcohol, respectively. As shown in this figure, the rise of the absorbance of the hydrated electron is very fast, while the rise time depends on the number of the carbon in the alkyl group and/or the structure of the individual alcohol molecule. Since the formation time of the hydrated electron is known to be very short $\left(0.2 \mathrm{ps}^{10},<0.3 \mathrm{ps}^{8}\right)$, the rise time of the present observation for $\mathrm{H}_{2} \mathrm{O}$ may be determined by the pulse width of two-photon excitation and the monitoring continuum. By using the value of $20 \mathrm{ps}$ for excitation pulse width and $22 \mathrm{ps}$ for the monitoring pulse width, and assuming a simultaneous biphotonic process for ionization, we have simulated the rise curves of the absorbance of the solvated electron with its formation time $\tau_{\mathrm{s}}$ as parameter. As Figure 4 shows, the rise curve of the hydrated electron was reproduced very well with $\tau_{\mathrm{s}}=0$, indicating that the formation time is so short that the rise time of the absorbance corresponds to the response function of our apparatus. The formation times of the solvated electron obtained by such simulation in each liquid are presented in Table I. The solvation time of the electron measured by means of the picosecond pulse radiolysis in pure liquids ${ }^{6,7}$ and those obtained by picosecond laser two-photon ionization of the guest molecules in these solvents ${ }^{8-11}$ are also listed in this table. The excess kinetic energy of the electron immediately after

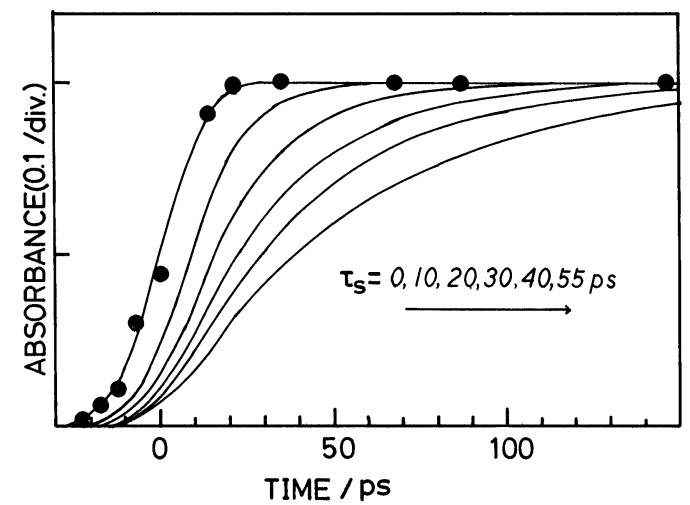

Figure 4 Simulated and observed rise curves of the hydrated electron: $(0)$, observed value and (---), simulated curve calculated with various values of formation time $\tau_{\mathrm{s}}$ as parameter. 
Table I Formation time of solvated electron in alcohol and in water.

\begin{tabular}{|c|c|c|c|c|}
\hline Liquid & $\begin{array}{l}\text { Dielectric } \\
\text { constant }\end{array}$ & $\begin{array}{l}\text { Picosecond 266-nm } \\
\text { two-photon } \\
\text { photolysis: } \\
\text { present results } \\
\text { (pure liquids) } \\
\tau_{s}(\mathrm{ps})\end{array}$ & $\begin{array}{l}\text { Picosecond } \\
\text { radiolysis } \\
\text { (pure liquid) } \\
\tau_{\mathrm{s}}(\mathrm{ps}) \dagger\end{array}$ & $\begin{array}{l}\text { Picosecond } \\
\text { laser photolysis } \\
\text { (photoionizatior } \\
\text { of solute) } \\
\tau_{\mathrm{s}}(\mathrm{ps}) \dagger\end{array}$ \\
\hline $\begin{array}{l}\mathrm{H}_{2} \mathrm{O} \\
\text { Methanol } \\
\text { Ethanol } \\
\text { 1-Propanol } \\
\text { 2-Propanol } \\
\text { 1-Butanol } \\
\text { 1-Pentanol } \\
\text { tert-Butyl alcohol } \\
\text { Ethylene glycol }\end{array}$ & $\begin{array}{l}78.8 \\
32.7 \\
24.6 \\
20.3 \\
19.9 \\
17.5 \\
13.9 \\
12.5 \\
37.7\end{array}$ & $\begin{array}{l}<3 \\
<10 \\
18 \pm 2 \\
22 \pm 2 \\
23 \pm 2 \\
21 \pm 4 \\
22 \pm 6 \\
45 \pm 5 \\
<10\end{array}$ & $\begin{array}{l}<3^{4} \\
10^{6}, 10.7 \pm 1^{4} \\
18^{6}, 23 \pm 2^{4} \\
24^{6}, 34 \pm 3^{4} \\
25^{6} \\
30^{6}, 39 \pm 5^{4} \\
34^{6} \\
54^{6} \\
<5^{6}\end{array}$ & $\begin{array}{l}<0.3^{8}, 0.2^{10} \\
17 \pm 3^{7} \\
26 \pm 2^{7}, \leqslant 20^{9} \\
\sim 20^{9} \\
30 \pm 3^{9}\end{array}$ \\
\hline
\end{tabular}

$\dagger$ Superscript numbers indicate the literature source of the data.

ejection depends on the excitation energy, the ionization potential and the mechanism of ionization. The distribution of the distance between the electron and the parent cation after thermalization may be affected by the difference of the excess kinetic energy. Furthermore, the electron ejected by electron pulse irradiation is solvated in the spurs where the positive ion and electron distributions can be quite different from those in the picosecond laser photoionization. On the other hand, in the solution containing the relatively large solute molecules, the solvation shell formed around the parent cation in the course of ionization might cause a change in the liquid structure.

However, the results in Table I indicate that the formation time of the solvated electron does not show drastic differences, depending on the excess energy difference and on the presence of guest solute molecules. Moreover, the solvated electron observed at delay times longer than a few tens of picoseconds is considered to be produced via the same precursor species on the basis of its spectral evolution in all cases examined by the different methods described above. The spectral shift from the infrared (IR) to the visible region corresponding to the solvated electron formation, as we have observed in Figure 2, was frequently confirmed in the radiolysis study ${ }^{6,7}$. This spectral shift has been interpreted as the gradual change from a shallow trapped elec- 
tron, of which the absorption maximum is in the IR region, to the solvated electron whose absorption maximum is in the visible region. The rotation of solvent molecules has been considered to play a major role in this process because of the good correlation of the formation time of solvated electrons with the dielectric relaxation time, $\tau_{2}$ (for the rotation of monomer in the solvent ${ }^{5-7}$ ). Also, in the experiments of the picosecond two-photon ionization of solutes where the measurements were performed only at a few isolated wavelengths, the rise of the absorbance in the visible region, together with the decrease in the IR region, has been confirmed ${ }^{8,10}$. Summarizing the above discussions on formation time and spectral shift of the solvated electron, it may be concluded that the solvated electron is produced from the same precursor species in all cases discussed above, the so-called trapped electron, and the difference of the excitation source conditions and the presence of the relatively large solute does not seem to change this essential pathway.

More detailed inspections of the formation times obtained by various methods indicate the existence of some effects of excess energy and liquid structure on the solvation process as discussed below. Comparison of our present results with those obtained by radiolysis appears to give information about the excess energy dependence of the solvation process. The present values of the solvation times seem to be a little shorter than those obtained by radiolysis, especially in liquids with a relatively small dielectric constant. This seems to be related to the results of our recent investigation on the geminate ion-pair in pure liquid cyclohexane produced via a simultaneous two-photon absorption of 266-nm laser pulse ${ }^{16}$.

According to our analysis, the geminate electron locates at 30-40 $\AA$, or $0.1-0.15 r_{\mathrm{c}}$, apart from the parent cation, where $r_{\mathrm{c}}$ is the Onsager length, and the distribution function for this electron can be approximated by a delta function, suggesting a very narrow distribution. Contrary to this, it has been reported ${ }^{17}$ that, in the case of the geminate pair produced by means of the $\beta$-ray irradiation of liquid cyclohexane, the distribution can be described by an exponential function of the electron-parent cation distance and the distribution maximum is around $60 \AA, 0.22 r_{\mathrm{c}}$. Although this result on the non-polar liquid may not be directly applicable to the present results in the polar liquids because the coulombic interaction between two charged particles is not so effective in a polar medium as that in a non-polar solvent, the 
trend that the $\beta$-ray excitation produces electron distribution in a wider distance range and that the distribution maximum is further from the parent cation than that obtained by the 266-nm two-photon excitation, seems to be probable also in the present case, especially in less polar liquids. Actually, as stated above, the formation time of the solvated electron obtained in the present work is a little shorter than the corresponding one obtained by pulse radiolysis, in general, especially in the case of alcohols with smaller dielectric constants.

There are not sufficient data for the elucidation of the effect of the presence of relatively large solute molecules, except for some cases with water. Nevertheless, we can see that the solvation times of an electron ejected via a two-photon ionization of pyrene with third harmonics using a $\mathrm{Nd}^{3+}$ glass laser in methanol and ethanol ${ }^{8}$, are a little longer than values obtained by other methods in the same alcohols. Contrary to this, the solvation times of ejected electrons from TMPD $\left(\mathrm{N}, \mathrm{N}, \mathrm{N}^{\prime}, \mathrm{N}^{\prime}\right.$-tetramethyl-p-phenylenediamine) in the same alcohols with the same exciting light source ${ }^{10}$ are very similar to those obtained in pure liquids. These differences might be explained as follows. The degree of destruction of the liquid structure near the site of solvated electron formation seems to depend on the size of the solvation sphere of the parent cation and that of the solvated electron. Therefore, the distance of the parent cation and the thermalized electron as discussed above also play an important role in this case. The electron ejected from a solute with low ionization threshold has a higher kinetic energy than that ejected from a solute with a high ionization threshold, if the excitation energy is the same. If this difference results in the difference of the distance between the solvated electron and the parent cation, the formation time of the solvated electron may be affected by this. The liquid structure around the electron at a distance from the solvation sphere of the parent cation may be almost the same as in the pure liquid, while in the vicinity of the solvated parent cation, the electron solvation process will require some rearrangements of the cation solvation shell. Hence, with increase of the excitation energy and/or with decrease of the ionization potential of the solute molecule, the deviation of the liquid structure from that in the pure liquid would become smaller. The ionization threshold of TMPD is known to be much lower than that of pyrene in a polar liquid and it was found that the ionization occurs even in the course of the relaxation from the fluorescent state ${ }^{18}$. 


\section{SUMMARY}

As discussed above, the present picosecond 266-nm two-photon photolysis provides an ingenious approach to the solvation process of the electron in polar liquids under homogeneous conditions. Comparison of the results obtained by the present method with those by other methods gives important information on the solvation dynamics, the mechanisms of electron thermalization, and consequent distribution function of distance with respect to the electron and the geminate cation. Further enhancement of the time resolution of the investigation in the multiphoton photolysis as well as pulse radiolysis studies will be very important for the elucidation of the formation dynamics of the solvated electron.

\section{Acknowledgement}

This work was partly supported by Grants-in-Aid (No. 5843003, No. 59045097) from the Japanese Ministry of Education, Science and Culture to NM.

\section{References}

1. H. Masuhara, H. Miyasaka, N. Ikeda and N. Mataga, Chem. Phys. Lett. 82, 59 (1981).

2. H. Miyasaka, H. Masuhara and N. Mataga, J. Phys. Chem. 89, 1631 (1985).

3. H. Miyasaka, H. Masuhara and N. Mataga, Chem. Phys. Lett. 118, 459 (1985).

4. H. Miyasaka and N. Mataga, Chem. Phys. Lett. 126, 219 (1986).

5. W. J. Chase and J. W. Hunt, J. Phys. Chem. 79, 2835 (1975).

6. G. E. Hall and G. A. Kenney-Wallace, Chem. Phys. 32, 313 (1978).

7. G. A. Kenney-Wallace and C. D. Jonah, J. Phys. Chem. 86, 2572 (1982).

8. Y. Wang, M. K. Crawford, M. J. McAuliffe and K. B. Eisenthal, Chem. Phys. Lett. 74, $160(1980)$.

9. J. M. Wiesenfeld and E. P. Ippen, Chem. Phys. Lett. 73, 47 (1980).

10. D. Huppert, G. A. Kenney-Wallace and P. M. Rentzepis, J. Chem. Phys. 75, 2265 (1981).

11. Y. Gauduel, A. Migus, J. L. Martin and A. Antonetti, Chem. Phys. Lett. 108, 319 (1984).

12. H. Miyasaka, H. Masuhara and N. Mataga, Chem. Phys. Lett. 98, 277 (1983).

13. H. Masuhara, N. Ikeda, H. Miyasaka and N. Mataga, J. Spectros. Soc. Japan 31, 19 (1982).

14. H. Miyasaka, H. Masuhara and N. Mataga, Laser Chem. 1, 357 (1983).

15. H. Masuhara, H. Miyasaka, A. Karen, T. Uemiya, N. Mataga, M. Koishi, A. Takeshima and Y. Tsuchiya. Opt. Commun. 44, 426 (1983).

16. H. Miyasaka and N. Mataga, Chem. Phys. Lett. in press.

17. H. T. Choi, J. A. Haglund and S. Lipsky, J. Phys. Chem. 87, 1583 (1983).

18. Y. Hirata and N. Mataga, J. Phys. Chem. 87, 3190 (1983). 